\title{
Influencia del filtro afectivo en el desarrollo de la producción oral del aprendizaje del idioma inglés
}

Fecha de recepción : 02-02-2021 • Fecha de aceptación: 01-04-2021 • Fecha de publicación: 10-05-2021

\author{
Nancy Cristina Uquillas Jaramillo' \\ Universidad de las Fuerzas Armadas ESPE, Ecuador \\ ncuquillas@espe.edu.ec \\ https://orcid.org/0000-0001-9829-5625
}

Karen Stephany Córdova Vera ${ }^{2}$

Universidad de las Fuerzas Armadas ESPE, Ecuador

kscordova@espe.edu.ec

https://orcid.org/0000-0001-7986-0430

\section{Resumen}

En la actualidad existen varias técnicas en el proceso de enseñanza - aprendizaje general y en torno a la adquisición de un segundo idioma; sin embargo, el hablar del filtro afectivo aún constituye un mito, pues se ha comprobado en investigaciones que el estudiante requiere no solo aprender los procesos convencionales de aprendizaje, sino qué, para la adquisición de un segundo idioma, la persona debe mantenerse motivada, con autoconfianza y sin ansiedad, a fin de que el aprendizaje sea dinámico y no rígido, tal como sucede con la adquisición del idioma natal. La información recopilada se basa en una investigación descriptiva-bibliográfica, para adentrarse a las opiniones e investigaciones de autores sobre esta temática de la influencia del filtro afectivo en el desarrollo, principalmente de la producción oral en el aprendizaje del inglés, donde se obtuvo que, a mayor coerción afectiva del estudiante, mejor será su afianzamiento en el aprendizaje del idioma inglés, y mucho más en lo que se refiere a la producción oral.

Palabras clave: afecto, filtro, adquisición, inglés, motivación, aprendizaje. 


\begin{abstract}
Currently there are many techniques in the general teaching-learning process and around the acquisition of a second language, however, talking about the affective filter still constitutes a myth, since the student has been proven in research that it requires not only learning conventional learning processes, because for the acquisition of a second language, the person must remain motivated, with self-confidence and without anxiety, so that learning is dynamic and not rigid, as happens with the acquisition of the native language, for which Information has been compiled based on a descriptive-bibliographic research, to delve into the opinions and research of authors on this issue of the influence of the affective filter in the development mainly of oral production in learning English, where it was obtained that, The greater the affective coercion of the student, the better his / her consolidation in learning the English language, and much more what refers to the oral production.
\end{abstract}

Keywords: affective filter, acquisition, english, motivation, learning. 


\section{Introducción}

Cuando se habla de educación hay que referirse al proceso en el que se comunican conocimientos, valores, costumbres y formas de actuar. La educación es el proceso por el cual, el ser humano, aprende diversas materias inherentes a él (Abreu, Gallegos, Jácome, \& Martínez, 2017). Esta está presente en todas las acciones, sentimientos y actitudes, y también comparte ideas, cultura, conocimientos, entre otros. Por esta razón, no puede dejarse de lado el proceso de enseñanzaaprendizaje, pues son los educadores y los educandos quienes se encuentran involucrados en el proceso.

Mientras que las formas menores de aprendizaje, según Schunk (1997, citado por Aguilera, 2004) pueden ocurrir en su ausencia, la motivación cumple una función importante en el aprendizaje. Este autor afirma que los estudiantes motivados para aprender, prestan atención a la enseñanza, se dedican a repasar la información relacionada con sus conocimientos y hacen preguntas. Por esto, puede afirmarse que para aprender sólo hace falta suficiente motivación, ya que existen diferentes tipos de aprendizaje y no todos ellos se concretan a un aula de clase.

Si el aprendizaje es un proceso en el que la motivación debe estar presente, y para que éste sea significativo y logre perdurar en el tiempo se requiere que la persona se comprometa a relacionar sus conocimientos con los que está adquiriendo; entonces el modelo de enseñanza que se pretenda implementar cobrará mayor importancia. Según Gallo y Lozano (2000), el aprendizaje que sólo depende de la inteligencia del alumno ha pasado a la historia. Actualmente variables como la atención y la motivación son imprescindibles y se ha dejado atrás el aprendizaje exclusivamente memorístico.

La educación que se proponga a la formación de la personalidad integral del educando debe tomar en consideración dos grandes dimensiones: la cognitiva y la emotiva. El desarrollo cognitivo ha recibido tradicionalmente un énfasis especial en menoscabo del desarrollo emocional, el cual ha quedado prácticamente olvidado en el escenario educativo. Una educación que se limita a los aspectos cognitivos deja fuera aspectos importantes del desarrollo integral. Por esta razón se presentan dificultades de aprendizaje, índices elevados de aplazados, estrés ante los exámenes, altos niveles de ansiedad que bloquean el aprendizaje y otros fenómenos relacionados con el fracaso escolar.

Del mismo modo, el sistema educativo se limita a desarrollar, y sólo en parte, la inteligencia cognitiva; pues se concibe el potencial académico como sinónimo de habilidades lingüístico verbales, siendo la gran mayoría de éstas confundidas generalmente con las capacidades memorísticas del educando en torno a cualquier tema; sin tomar en cuenta los procesos emocionales, como el gusto o la empatía que en el alumno logre la materia de estudio. Por ello, no se puede obviar el alto grado de desmotivación de los alumnos en el aula de clase (la baja motivación del estudiantado es uno de los problemas actuales de la educación); especialmente en la lengua extranjera que se caracteriza por no ser del agrado de la mayoría de los educandos, teniendo sentimientos de miedo y tensión hacia la mencionada materia, sobre todo cuando se trata de la habilidad oral. El reto que se abre en el marco de la educación emocional es motivar 
a partir de las emociones, estas desencadenan una acción, por tanto, constituye una motivación para hacer algo. Emoción y motivación están interrelacionadas, tanto que la etimología de ambas palabras es la misma, mover.

A su vez, existe una gran preocupación docente por desarrollar en el alumno su inteligencia cognitiva, sustentándose en el hecho que de esta manera indudablemente alcanzará el éxito educativo, cuando por el contrario, las hoy llamadas capacidades blandas (inteligencia emocional), pueden ser aún más fundamentales para el éxito en la vida. Una educación limitada a los aspectos cognitivos deja fuera aspectos importantes del desarrollo; puesto que, en este afán de sólo desarrollar el cociente intelectual de los jóvenes, se soslayan las capacidades emocionales, las cuales parecen estar disminuyendo vertiginosamente.

Lo ideal sería que las capacidades cognitivas interactuaran de forma dinámica con las capacidades emocionales, por cuanto éstas no se oponen, sino que se complementan; entonces, sería posible que una persona pudiera destacarse tanto en una como en la otra y, por consiguiente, puede lograr el equilibrio requerido para el verdadero éxito educativo. Por esta razón, el individuo debe ligarse afectivamente en la experiencia de aprender; debe estar motivado, es decir, experimentar el deseo de aprender, sentir que puede hacerlo, pues sólo así, se despertará en él la necesidad suficiente para poner en funcionamiento su potencial cerebral.

Las diferentes teorías sobre el logro del aprendizaje tienen que ver con el estudio y el enfoque dado, así se propone orientarse al rol que desempeñan los softwares educativos para el presente proyecto. Según Valenzuela et al. (2018) "Ios aportes de cada teoría no son necesariamente convergentes como no lo es la perspectiva desde la cual se analiza el fenómeno de cada caso, ni los métodos usados para obtener el conocimiento."; es decir, la teoría que deba aplicarse para el proceso de enseñanza-aprendizaje tiene que ver con el ámbito en el que se va a ocupar y el fenómeno que se ha de estudiar.

Además, es dable que surja la necesidad de que cada aporte esté relacionado con el tema a tratar, por este motivo, se presenta una breve descripción de las características de las principales teorías de enseñanza-aprendizaje, de la cual se toma como ejemplo al aprendizaje significativo según Piaget para la aplicación del presente estudio.

De acuerdo a lo anterior, es relevante conocer las estrategias de enseñanza y aprendizaje para asegurar una praxis pedagógica con intencionalidad al logro de metas en la formación práctica y reflexiva del estudiante. En función de lo expresado, cabe decir que el estudiante, mediante las estrategias, tanto de enseñanza, como de aprendizaje, le es posible una participación más dinámica; es decir, tiene mayores oportunidades para confrontar la variada gama de interpretaciones que ya posee y que es capaz de mejorar, actualizar, compartir o modificar a través de la actuación de su conciencia y de su pensamiento lógico. Esto conlleva entonces a que el estudiante deje de ser objeto, para convertirse en sujeto de su propia educación.

Es importante mencionar que, entre las lenguas del mundo, el chino mandarín, el hindú, el inglés y el español ocupan las primeras posiciones por la importancia mundial y el número de hablantes. De éstas, el mandarín y el hindú son propiamente lenguas nacionales, aun cuando su alcance 
geográfico ha crecido al ritmo de la migración, y representan ante todo las visiones particulares de culturas milenarias. El inglés, en cambio, se ha transformado en una lengua universal, en el idioma de la globalización y de la expansión planetaria de la cultura y los valores occidentales, es el idioma internacional por excelencia. Actualmente más de 400 millones de personas tienen al inglés como lengua materna, 300 millones más lo hablan como segunda lengua, y entre 500 y 750 millones poseen ciertos rudimentos del idioma (Gutiérrez y Landeros, 2010).

El predominio del inglés no se computa sólo en números; la influencia económica, política, científica, tecnológica y cultural de los Estados Unidos ha sido influenciada bajo la lengua inglesa, la cual se convirtió en la lengua franca de la diplomacia, del comercio internacional, de las telecomunicaciones, Internet, cultura de masas y debates académicos. Sus terminologías y su sintaxis han aportado a todas las lenguas del mundo, sus referencias culturales se han fijado en la mente de millones. El inglés se ha vuelto, en la acepción más amplia del término, una lengua imperial (Cancino, 2006).

Por lo tanto, el desarrollo de la competencia comunicativa en una o más lenguas extranjeras para posibilitar la participación en igualdad de condiciones, en la cultura global sin perder el sentido de pertenencia a nuestra cultura, es parte de esa educación integral. En consecuencia, se estima necesario asegurar el conocimiento y la habilidad de comunicación en un segundo idioma, fundamentalmente el idioma inglés, por cuanto se ha convertido en una verdadera lengua internacional, utilizada por muchos sin importar sus diferencias geográficas o ideológicas (Martínez, 2018).

Por ello es que dentro de estos factores se incluye aquellos aspectos emocionales, puesto que se conoce que las estrategias afectivas influirán también en el dominio de una segunda lengua, ya que "son factores psicológicos naturales que tienden a influir negativamente cuando no se les proporciona docentes preparados, o metodologías acordes con el nivel y la realidad de los estudiantes, así como se genera la gran necesidad de un aprendizaje humanista" (Moreno, 2017).

De esta manera, la investigación emprendida nace de la preocupación por las debilidades de un grupo de estudiantes para poder expresarse de manera segura en lengua materna y en lengua extranjera. En un estudio minucioso, producto de intercambios pedagógicos, debates académicos y de la realidad observada, se determinó las debilidades de los estudiantes para poder organizar sus pensamientos en una lengua extranjera y poder expresarlos sin que esto produzca niveles de ansiedad; además, se observó que los estudiantes no sienten al aula de clases como un ambiente seguro en el cual los errores sean aceptados y asumidos como un momento de aprendizaje, por el contrario, los errores en la pronunciación se vuelven momentos de crítica o burla.

De igual forma, se demostró que el desarrollo emocional puede ofrecer muchas ventajas para tener éxito en la escuela; tal vez lo más interesante es que los educadores pueden enseñar a sus alumnos formas de autocontrol, pero sin suprimir las emociones, como, por ejemplo: controlar la ansiedad por un examen y el gusto por alguna materia, porque la estabilidad emocional es fundamental para generar aprendizajes significativos. 
De esta manera, podremos indicar que el objetivo de esta investigación es demostrar que los factores afectivos como la motivación intrínseca y extrínseca, las emociones, actitudes y aun las creencias actúan como fuerzas impulsadoras para mejorar la producción oral en el proceso de la enseñanza-aprendizaje del idioma inglés. Además, pretende demostrar que los niveles del filtro afectivo que se maneja en clase dependen en gran parte de la motivación del docente y de cómo el estudiante perciba al aula de clases un lugar seguro para poder expresar sus ideas de manera oral, sin que esto cause ningún tipo de críticas, burlas o correcciones permanentes públicas por parte del docente y en caso de ser así, cómo estos comportamientos influyen en la producción oral.

\subsection{La motivación como recurso socio afectivo para el aprendizaje de una segunda lengua}

"La falta de motivación es uno de los factores principales que se reflejan en el nivel del filtro afectivo de los aprendices" (Shehri, 2012), por otro lado "es posible que los aspectos psicológicos generen influencia en la adquisición de una lengua extranjera" (Sánchez, 2017), siendo uno de los aspectos más analizados como factores de riesgo los nervios, el estrés, tartamudeo, y otras problemáticas que se generan al momento de las exigencias totalmente rígidas que tienen los estudiantes para la producción oral del idioma extranjero.

Sin embargo, aún el docente, en ciertos casos, se sigue presentando como el conocedor, como quien debe llenar al estudiante de ellos para lograr un correcto aprendizaje del idioma extranjero cuando realmente las cosas son distintas, ya que se deja de lado el ente emocional, aquel que lleva al estudiante a generar motivación, pues por ejemplo, Montes de Oca (2017), determina que "el juicio que tienen los estudiantes acerca de sí mismos son determinantes en la construcción de la personalidad y en el deseo por aprender", es decir, que situaciones como el autoestima, el afianzamiento o confianza en sí mismo, son valores que los docentes de idioma extranjero han de trabajar para realmente profundizar el aprendizaje, y permitir que el estudiante se motive por adquirir el lenguaje no por obligación, sino por plena motivación intrínseca y el deseo de superación.

\subsection{La motivación como estrategia}

A menudo se asume que la motivación va de la mano con el inicio de una actividad de aprendizaje y que una vez activada continúa automáticamente hacia el final. Desde la perspectiva constructivista se piensa que la motivación no se activa de manera automática, ni es privativa del inicio de la actividad o tarea, sino que abarca todo el episodio de enseñanza-aprendizaje y que el alumno, así como el docente, deben realizar deliberadamente ciertas acciones, antes, durante y al final, para que persista o se incremente una disposición favorable para el estudio.

Parra (2014) señala que mediante la categoría motivación del contenido se identifica aquella etapa del proceso en la cual se presenta el objeto a los estudiantes, promoviendo con ello su acercamiento e interés por el contenido a partir del objeto. En esta etapa la acción del profesor es fundamental, pues es quien le presenta al estudiante el objeto y el contenido preferentemente como un problema que crea una necesidad de búsqueda de información, promoviendo así la motivación de los estudiantes. 
Para Ospina (2006) son dos las condiciones que deben darse para producir en un individuo la motivación intrínseca hacia la realización de una tarea (De Charms, 1984; en Alonso, 1991; Brophy, 1998):

- Que la realización de la tarea sea ocasión para percibir o experimentar que se es competente.

- Que se dé la experiencia de autonomía, que el sujeto sienta que ejerce control sobre su entorno y su propia conducta.

Esto significa en relación con el manejo del entorno que las opciones de acción y el número de alternativas para el alumno sean lo más numerosas posible. Además, implica que el alumno necesita tomar consciencia de sus propias motivaciones y ser sensible a la autonomía de los demás; al mismo tiempo debe comprender el significado de la satisfacción interna del aprendizaje y qué puede hacer a manera de incrementar su autonomía.

Según Ospina (2006), para motivar intrínsecamente a los alumnos se requiere lograr:

- Que den más valor al hecho de aprender que al de tener éxito o fracaso.

- Que consideren a la inteligencia y a las habilidades de estudio como algo modificable y no como inmutables.

- Que centren más su atención en la experiencia de aprender que en las recompensas externas.

- Facilitar su autonomía y control por medio de mostrar la relevancia y significatividad de las tareas (p. 85).

En la motivación para el aprendizaje, como en general en la motivación humana, influyen múltiples factores. La influencia de estos factores no se produce mecánicamente, de tal manera que pudiera predecirse su efecto o ser manipulados para conseguir el efecto deseado.

Para García (2012) los factores que influyen en la motivación pueden clasificarse siguiendo diversos criterios, pero lo más lógico es fijarse en si dependen fundamentalmente de la propia persona (factores personales) o más bien se deben al contexto que envuelven al sujeto e influyen en él (familia, sociedad, profesores, centro educativo, entre otros).

\subsection{La autoestima como factor predominante en el aprendizaje de una segunda lengua}

En investigaciones sociolingüísticas realizadas se señala que el aprendizaje del inglés es el producto del proceso de enseñanza-aprendizaje en donde intervienen variables psicológicas y sociales propias de los estudiantes, profesores y del contexto institucional, que entran en interacción e interdependencia en la misión psicopedagógica de instrucción y formación humana. Una de las variables propias del estudiante es la autoestima, considerada como el aprecio que la persona refleja hacia sí mismo, en base a sus logros y fracasos, es por ello que constituye una variable importante para el éxito o fracaso del sujeto en cualquier actividad que se desempeñe, 
quedando de manera fija en la psiquis del sujeto la forma en que se resuelven los problemas que se le presentan en lo cotidiano.

Conviene en primer lugar considerar la variable autoestima desde varios aportes teóricos y enfoques psicológicos y pedagógicos de reconocidos autores:

Para Lojano (2017, citado en Gades, 1998), esta es entendida como la experiencia fundamental de que podemos llevar una vida significativa y cumplir sus exigencias. Más concretamente, podemos decir que es la confianza en nuestra capacidad de pensar, es nuestra capacidad de enfrentarnos a los desafíos básicos de la vida, en nuestro derecho a triunfar y a ser felices. "La esencia de la autoestima es confiar en la propia mente y en saber que somos merecedores de la felicidad", agrega (p. 32-33).

Es importante tener confianza en nosotros mismos para desenvolvernos en el diario, en esto influyen los mensajes recibidos en la infancia, que van dejando su huella a lo largo de la vida; y son estas experiencias que ayudan a desarrollar la autoestima. De esto depende la calidad y los tipos de autoestima que se tenga, la confianza que le dará a la persona, capacidad de salir adelante, triunfar cuando se lo propone, o la posibilidad de ser victorioso cuando así lo quiere. Por ejemplo: si a un estudiante se le repite varias veces que es torpe e incapaz de hacer las cosas bien, tendrá siempre este mensaje en su mente, es decir, tendría estos pensamientos automáticos y le impedirán que logre su objetivo, porque no tiene confianza en el mismo, ni en sus capacidades para lograr su meta.

Al respecto, Ausubel lo afirma citado en Plan de Acción Tutorial Gades (1998):

La adquisición de nuevas ideas y aprendizajes está subordinada a nuestras actitudes básicas, de ellas depende que se generen energías más intensas de atención y concentración. Aquí reside en buena medida la causa del fracaso escolar. La autoestima es la suma de la confianza y el respeto que debemos sentir por nosotros mismos y refleja el juicio de valor que cada uno hace de su persona para enfrentarse a los desafíos que presenta nuestra existencia. La autoestima es la visión más profunda que cada cual tiene de sí mismos, es la aceptación positiva de la propia identidad y se sustenta en el concepto de nuestra valía personal y de nuestra capacidad (p. 30).

Por otra parte, el filtro afectivo en el aprendizaje oral del idioma inglés puede ser potencializado por herramientas como la música, videos o situaciones de la vida real que pueden ser afianzadas para adquirir el idioma inglés, adicional el ambiente positivo que se desarrolle en el aula, un ambiente que permita el error y lo utilice como momento de aprendizaje.

En consecuencia, los pensamientos y creencias hacia el propio yo del estudiante determinan los sentimientos y el nivel de autoestima de cada ser humano en general y cada estudiante en particular. Cuando un alumno tiene excelentes calificaciones, tendrá una autoestima alta, su auto concepto es adecuado, los mensajes que ha recibido desde su infancia ha fortalecido su autoestima. Podemos darnos cuenta que alumnos que tienen un bajo rendimiento también tiene una autoestima baja porque la construcción de la autoestima no ha sido la correcta, el entorno 
que se desenvuelve contribuye al aumento o disminución, los mensajes positivos y negativos, la confianza o desconfianza determinan el nivel de autoestima y por ende el rendimiento escolar.

\subsection{Etapas de un correcto aprendizaje lúdico}

- La escucha: según lo dicho por Parra (2014), "el interés se da con la estimulación por aprender, con lo que los pensamientos de la persona se profundizan porque esta está abierta y mantiene la intención de conocer o descubrir de cerca una determinada situación u objeto" (p.42).

En esta instancia, la escucha es parte de la comunicación humana, según el autor, "es una interacción entre medios de tipo orales y escritos, que permiten comunicarse y codificar la información, así como comprenderla e interpretarla" (p.14). La escucha es la puerta de entrada para que el estudiante relacione un objeto con su pronunciación, pues en su mente se graban los códigos preestablecidos para el lenguaje.

- Adquisición: en esta etapa, el conocimiento "es un proceso que inicia con la visualización de los contenidos de la asignatura; sin embargo, en algunos casos, solo cuando se vive estos contenidos es posible lograr una idea concreta de los mismos" (Yánez, 2015).

Es posible evidenciar que los conocimientos se adquieren con un aprendizaje efectivo, en torno a la asimilación de lo vivido, lo que incide también en el desarrollo afectivo del estudiante porque integra acción con emoción.

"El estudiante olvida un hecho en conflicto y prefiere pensar en hecho de confianza, lo que se retiene son entonces ideas básicas de lo que es verdadero y razonable" (Ausubel, 2002).

- Repetición y almacenamiento: de acuerdo a lo indicado por Sáiz (2018) el almacenamiento y repetición de la información "son las actividades que permiten retener y generar una memoria a corto plazo o largo plazo con estrategias como copiar, repetición, recursos nemotécnicos".

Entre las estrategias más utilizadas que ayudan a la repetición se encuentra el almacenamiento: "reciclaje de información para mantenerla en la memoria a corto plazo" (Buey, 1999).

Ejemplos de este tipo de estrategia está el repetir un número de teléfono, el aprender la tabla periódica de los elementos químicos, entre otras situaciones que requieren de una memoria de almacenamiento temporal, y por ende, también se asume la escucha de una canción por repetidas veces, siendo un método adecuado de afianzamiento cognitivo y asociativo.

- Retención y asimilación: en esta etapa de aprendizaje se plantea ya un ente de retención mediante la abstracción, por ello es que aquí el estudiante ocupa la capacidad analítica, por supuesto que esta se difunde cuando está motivado y abierto a aprender. En esta instancia también interviene "la capacidad crítica y analítica del estudiante debido a que este debe asimilar el contenido y los debe discriminar para que de esta forma se logre generar sus propios conceptos" (Díaz, 2011). 
"Para verificar que existe la comprensión asertiva de conocimientos es necesario hacer que el estudiante lo practique en algún caso específico, logrando la comprensión no solo de forma teórica sino práctica" (Yánez, 2015). Esta comprensión obliga a profundizar los conocimientos y convertirlos en abstracciones de la realidad, así como el estudiante tiende a separar una fase de aprendizaje con otra.

\subsection{Aspectos importantes donde influye el desarrollo afectivo para el aprendizaje del segundo idioma}

Es necesario que los docentes creen una relación armónica con el estudiante, pero sobre todo enfocada en generar un aprendizaje constructivo en torno al idioma inglés, para lo cual se requieren de estrategias específicas, las cuales el docente ha de fomentar a generar un nexo entre afecto y adquisición del idioma.

Como manifiesta Nathaniel Brande (1994), como sucede con los padres, a un maestro le es más fácil inspirar la autoestima en los estudiantes si el maestro ejemplifica y sirve de modelo de un sentido de la identidad sano y afirmativo. En realidad, algunas investigaciones sugieren que éste es el factor primordial de la capacidad del maestro para fomentar la autoestima de un estudiante.

Es decir que, el docente debe empezar por mantener una autoestima correcta, pero a su vez, debe lograr transmitir esto a sus estudiantes para librarlos de la ansiedad y fomentar su autoconfianza, es así que para que el filtro afectivo sea motivado, según Cassany (2008) "los estudiantes que están motivados por aprender una lengua tienen un filtro afectivo bajo, pues sus estados de angustia, inestabilidad emocional, conflictos internos no facilitan la adquisición del conocimiento, lo que influye en el filtro afectivo es la actitud el docente con respecto a la clase" (p.53).

Entorno a adquirir el dominio de un idioma extranjero, se ha de desarrollar entonces:

- Comprensión auditiva (listening)

- Expresión oral (speaking)

- Comprensión de lectura (reading)

- Expresión escita (writing)

Por ello es que los aspectos influyentes para adquirir un idioma suelen relacionarse con "los factores afectivos en la lengua materna, la edad no le permite al niño sentir vergüenza por sus errores" (Cassany, 2018), pero cuando se adquiere el aprendizaje de la lengua extranjera produce ansiedad el no poder expresarse correctamente.

Por otra parte, se observa que el contexto de aprendizaje de la lengua materna posee un entorno natural, mientras que "en el caso de la lengua extranjera el estudiante está en un ambiente ficticio como lo es el aula de clase" (Cassany, 2018), por otra parte se conoce que "la lengua materna se la adquiere en la infancia, y por lo general el idioma extranjero se lo adquiere en edades escolares 
o adultas" (Cumbajín, 2018), así como "el desarrollo cognitivo del educando para la lengua materna va a la par con el desarrollo del lenguaje pero para la lengua extranjera es posterior a dicho desarrollo" (Díaz, 2011), todo lo anterior lleva a replantearse que se requiere la generación de herramientas propicias para acoplar al estudiante a un ambiente similar al materno, y para ello se requiere fomentar el desarrollo afectivo y cognitivo.

\subsection{Estrategias para fomentar el desarrollo afectivo para la adquisición del idioma inglés}

Entre las estrategias que permiten fomentar el desarrollo afectivo para la adquisición del idioma inglés están:

- Juegos

- Películas

- Música

- Otras actividades

Según la investigación, se indica que "la mayoría de estudiantes $(87,50 \%)$ que fueron encuestados afirman que se sienten mejor aprendiendo mediante juegos" (Cumbajín, 2018), esto quiere decir que se le toma al juego como una herramienta que supera al resto y que permite a los estudiantes del idioma inglés adquirir mayor empatía con este y afianzar la asociación entre afecto y adquisición del idioma.

\section{Metodología}

Se realizó la búsqueda de diferentes ensayos y estudios que cumplieron con los criterios de inclusión definidos para esta revisión en diferentes bases digitales, los cuales avalaron el requisito de explicar el mecanismo de acción del filtro afectivo a nivel de adquisición del idioma inglés. Otras fuentes fueron libros clásicos de psicología, pedagogía y ciencias cognitivas encontradas en bases de datos online como Google Académico, SciELO y Revistas indexadas en Scopus. Por ende, los términos de búsqueda utilizados fueron: "afecto", "filtro", "adquisición" "inglés" "motivación" "aprendizaje".

Para la selección de estudios se aplicaron los siguientes criterios de inclusión:

I. Estudios en idioma español e inglés.

II. Estudios en libros de pedagogía, repertorios, repositorios de pedagogía para un segundo idioma, diccionarios en español y de sinónimos y antónimos.

III. Se excluyeron de la revisión: estudios a los cuales no fue posible acceder en texto completo, fuentes de adquisición de segundo idioma que no tenía autores, sitios web no confiables. 


\section{Resultados}

Las emociones juegan un papel primordial en nuestras vidas. Por eso, no es extraño que hayan sido objeto de preocupaciones y análisis desde la antigüedad. Sin embargo, desde algunas teorías psicológicas se ha producido una perpleja resistencia hacia el estudio de las emociones, como mínimo hasta los años sesenta. El conductismo y el positivismo lógico consideraron que las emociones no podían ser objeto de investigación científica por no ser controlables y replicables.

No obstante, a partir de los años sesenta se va cambiando el rígido modelo conductista de estímulo-respuesta $(\mathrm{E}-\mathrm{R})$ por otro neoconductista de estímulo-organismo-respuesta (E-O-R), lo cual abre la puerta para el estudio de lo que sucede en la "caja negra" de la mente. La elaboración de constructos teóricos, no directamente observables, es una característica de las investigaciones que van aflorando a partir de ese momento. Paralelamente, adoptarse planteamientos holísticos, morales y sintéticos, con influencias de la teoría general de sistemas, tiene una mejor entrada el estudio de las emociones.

En esta época de cambio de tendencia se produce la llegada de la psicología cognitiva. Todo ello propicia la investigación de las emociones como una variable organísmica. Conviene recordar que la irrupción del paradigma cognitivo no supuso la entrada de las emociones en las investigaciones científicas. El "primer" cognitivismo o cognitivismo "frío", lejos de recuperar el estudio de los procesos emotivos y motivacionales, acentúo más si cabe su omisión. En los primeros modelos cognitivos, basados en el procesamiento de la información, llama la atención la ausencia de referencia a las emociones. Esta tendencia se verá agudizada en los modelos computacionales posteriores. Solamente ha mediado de los setenta, con el "segundo" cognitivismo, se abre la puerta al estudio de las emociones.

A partir de mediados de siglo, la psicología humanista presta una atención especial a las emociones. Posteriormente, la terapia cognitiva pone el énfasis en el control racional de las emociones. Se encuentran, por tanto, en estos pensadores, así como en muchos filósofos como Aristóteles, Kant, Spinoza, Hume, Bretano, Scheler, Bergson, Dewey, Heidegger, Sartre, Bedford Russell y muchos otros, teorizaciones sobres las emociones desde un planteamiento cognitivo. Es decir, el uso de conceptos cognitivos en el estudio de la emoción tiene una larga historia se remonta a la antigüedad y a la edad media.

Es en el marco del cognitivismo donde asistimos a un reciente despertar del interés por las emociones desde el punto de vista científico. Hasta los años sesenta ha prevalecido el enfoque analítico de las emociones disfóricas, sin llegar a un enfoque integrador que incluya las emociones positivas. A partir de entonces se empieza a prestar atención a las emociones, sobre todo desde la psicología humanista. Pero no es hasta finales de los ochenta cuando se produce un énfasis especial en las emociones, de tal forma que se pueda hablar de revolución emocional.

Durante la década de 1960 menudearon críticas por el hecho de que la educación no atendía al conjunto total de las capacidades de las personas: sólo atendía a lo cognitivo e ignoraba lo emocional. Como consecuencia se presenta el entusiasmo por introducir lo afectivo en las 
prácticas docentes, y es en la década de 1990 cuando se percibe un resurgimiento del interés por los temas emocionales.

El término "Inteligencia Emocional" fue utilizado por primera vez en 1990 por los psicólogos Salovey de la Universidad de Harward y Mayer de la Universidad de New Hasmpshire. Lo emplearon para describir las cualidades emocionales que parecen tener importancia para el éxito en la vida.

En 1990, Salovey y Mayer, (citado en Bueno, Teruel y Valero, 2005), fueron los primeros en definir cociente emocional como un "subconjunto de inteligencia social" que comprende la capacidad de controlar los sentimientos y emociones propios, así como de los demás, de discriminar entre ellos y utilizar la información para guiar los pensamientos y acciones. Se oponen al uso del término cociente emocional como sinónimo de Inteligencia Emocional, temiendo que lleve a la gente a pensar erróneamente, que existe un test preciso para medir el cociente emocional, incluso, que se pueda llegar a medir de alguna manera. De todos modos, subsiste el hecho que, aunque el coeficiente emocional nunca resulte medible, emerge de todas maneras como un concepto significativo. Aunque no se pueda medir con facilidad las emociones de los alumnos durante las clases de segunda lengua, por ejemplo: confianza en sí mismo, automotivación, autocontrol, empatía, entre otras, lo que sí resulta posible hacer es reconocer dichas capacidades emocionales en los alumnos y discutir sobre su importancia.

Según Mcleod (1992), (citado por Caballero, 2008):

El desarrollo de los estudios sobre cómo son los procesos de aprendizaje de los estudiantes y sus implicaciones en la práctica instruccional cada vez son más relevantes. Como hemos indicado, en esta última década se ha puesto énfasis especial en poner de manifiesto el papel de los factores afectivos en el aprendizaje.

Por su parte, en 1994, Mcleod (citado por Caballero, Cárdenas y Gómez, 2014), también expresó lo siguiente: "durante una larga época, los estudios sobre dimensión afectiva estuvieron reducidos al estudio de actitudes, en estas dos últimas décadas se ha ampliado al estudio de creencias y reacciones emocionales" (p. 29).

Por esta razón, es preciso desarrollar las capacidades emocionales dentro del aula de clases, por parte de los profesores y los alumnos, para así alcanzar el objetivo planteado y hacer del trabajo algo productivo. Al desarrollar la inteligencia emocional en las clases de inglés se podrán romper las barreras y temores que los educandos sienten por la citada asignatura, lo cual contribuye al crecimiento de la confianza y seguridad del aprendiz en el estudio de la misma. Porque no sirve de nada haber asimilado todos los conocimientos necesarios para presentar un examen, si entra en juego el miedo y provoca un bloqueo. Solamente en un ambiente equilibrado el alumno podrá incrementar la capacidad de perseverancia ante cualquier circunstancia adversa, por ejemplo, el fracaso ante un examen.

Según Vaello (2005): 
El manejo de las situaciones problemáticas exige de los profesores una gran cantidad de cualidades emocionales, tales como: Respeto por los alumnos, lo que impide ser hirientes incluso cuando están enfadados o en el trato con alumnos difíciles. La capacidad de manejar la propia indignación, un sentimiento de autoestima estable que le permita no convertir cada provocación de los alumnos en un ataque personal. El conocimiento de que el tono que emplea en el trato emocional de los mismos con los alumnos actúa sobre el desarrollo. La capacidad de ponerse en lugar de los alumnos y comprender sus motivos (empatía).

Básicamente se observa que en el aula de clase de inglés falta desarrollar la empatía, puesto que los profesores no se ponen en lugar de los alumnos. Esta actitud negativa se evidencia dentro del aula de clase por parte de los profesores que no se interesan por conocer la causa por la cual el alumno no comprende ciertos contenidos, sin tomar en cuenta que el conocer las necesidades e inquietudes de los alumnos es fundamental para alcanzar el éxito educativo. El hecho de que el docente esté emocionalmente apto contribuye a que el aula sea un lugar placentero para los estudiantes, puesto que los mismos serán respetados y, por consiguiente, construirán libremente los conocimientos de cualquier contenido, dejando atrás la ansiedad, el temor, la apatía y el desinterés hacia la citada asignatura.

En primer lugar, Parra (2014), presentó una propuesta de "Estrategias Creativas dirigidas al docente y el uso de la mediación en los procesos de enseñanza y aprendizaje", la iniciativa tuvo su origen en el intercambio continuo de ideas y experiencias de los docentes en el área de inglés, permitiendo de este modo realizar una investigación de campo en el escenario del aprendizaje significativo. Como resultado de la aplicación del instrumento a la muestra, se refleja la importancia del nivel de conocimiento que tiene los docentes en las concepciones teóricas y la aplicación práctica de estrategias innovadoras en fortalecer el aprendizaje significativo.

Por su parte, Fabregat (2015) realizaron un trabajo cuyo objetivo general fue: Diseñar unas estrategias para el desarrollo de la Inteligencia Emocional en pro de la formación de inglés de los alumnos, dicha investigación arrojó los siguientes resultados: La principal necesidad presente en clase de inglés, es la falta de un estudio sistemático por parte del docente sobre la materia emocional y de sus influencias afectivas en el conocimiento de la misma. Alumnos que tienen profesores inteligentes desde el punto de vista emocional, disfrutan más asistiendo a la escuela, aprenden sin pasar miedo alguno y van edificando una sana autoestima. Pero, sobre todo, la postura humana del profesor trasciende a ellos.

\section{Conclusiones}

Es de vital importancia entender que la adquisición y dominio de un segundo idioma amerita un proceso de aprendizaje constructivista, donde sea el mismo estudiante quien genere su propio conocimiento, integrándolo con la motivación y la mejora de autocontrol que se logra con procesos de afianzamiento del filtro afectivo.

Los procesos para el desarrollo cognitivo se fundamentan en la escucha, la adquisición, repetición y asimilación como producto de varias etapas y sub-etapas que se justifican al motivar al 
estudiante a adquirir un segundo idioma, entendiendo que si se fomenta el desarrollo afectivo será más factible cumplir con este proceso de forma concreta y encaminada al aprendizaje significativo.

Entre las estrategias que pueden generarse para mejorar el filtro afectivo para el aprendizaje de un segundo idioma como lo es el inglés se requieren algunas como música, videos, juegos y hoy en día las actividades interactivas mediante el uso de las TIC; sin embargo, en un estudio realizado en el Ecuador, determinó a los juegos como las herramientas fundamentales para mejorar o potencializar el desarrollo afectivo y por ende la correcta adquisición del idioma inglés.

Por esta razón, y dándole cumplimiento a las exigencias propias del sistema educativo, se recomienda diseñar talleres basados en los componentes de la inteligencia emocional. Proporcionando de esa manera estrategias, metodologías y técnicas que orienten el proceso didáctico, con el propósito de que los docentes, quienes demostraron a través de la información recabada con el instrumento no participar activamente en función de lograr un clima emocional, propicie las condiciones idóneas para que el alumno adquiera los conocimientos facilitados de forma significativa, se sumen así, a quienes por vocación o profesionalismo se esmeran por dar lo mejor de sí en pro de una formación integral.

Los componentes de la inteligencia emocional brindan las herramientas para lograr del proceso didáctico un resultado favorable, tanto para los docentes, como para los alumnos, porque así mismo como los alumnos mejoran el rendimiento académico y su actitud frente al proceso educativo, los docentes sentirán menos presión al notar que sus esfuerzos están generando excelentes resultados; creándose de esa manera un adecuado clima emocional.

Se requiere de un punto de referencia, en este caso se utilizaría la relación de notas, también se necesitará de un seguimiento, retroalimentación y evaluación constante que mantendrá informado acerca del desarrollo y progreso del proceso.

Se concluye resaltando que el coeficiente emocional puede ser desarrollado a cualquier edad, canalizando, planificando, coordinando los componentes de la inteligencia emocional para modificar la conducta indeseada, esto coadyuvará a generar un clima emocional adecuado. 


\section{Referencias}

Abreu, O., Gallegos, M., Jácome, J., \& Martínez, R. (2017). La Didáctica: Epistemología y Definición en la Facultad de Ciencias Administrativas y Económicas de la Universidad Técnica del Norte del Ecuador. Formación universitaria, 10(3). https://doi.org/10.4067/S0718-50062017000300009

Ausubel, D. (2002). Adquisición y retención del conocimiento, una perspectiva cognitiva. Barcelona: Paídos.

Branden, N., (1995), Los seis pilares de la autoestima, Barcelona: Paidós, ISBN: 978- 84-493-0144-5, p. 21-22

Buey, M. D. (1999). Fase de transformación de la información. Madrid.

Bueno G., Concepción \& Turuel M., María \& Valero S., Antonio. (2005). La Inteligencia Emocional en alumnos de Magisterio: La percepción y comprensión de los sentimientos y las emociones. https://www.redalyc.org/ pdf/274/27411927010.pdf

Caballero C., Ana, Blanco N., Lorenzo J, \& Guerrero B., Eloísa. (2008). El dominio afectivo en futuros maestros de matemáticas en la universidad de extremadura. Paradigma, 29(2), 157-171. http://ve.scielo.org/scielo. php?pid=S1011-22512008000200009\&script=sci arttext

Caballero C., Ana, \& Cárdenas L., Janeth, \& Gómez del Amo, Rosa (2014). El dominio afectivo en la resolución de problemas matemáticos: una jerarquización de sus descriptores. International Journal of Developmental and Educational Psychology, 7(1),233-246. $\quad$ https://doi.org/10.17060/ijodaep.2014.n1.v7.795

Cancino, R. (2006). El español - la empresa multinacional: El impacto de la lengua y cultura española en el mundo actual. Sociedad y Discurso (10). https://core.ac.uk/download/pdf/229016327.pdf

Cassany, D. (2018). Filtro afectivo. https://blogs-fcpolit.unr.edu.ar/programa/2008/03/09/filtro-afectivo-daniel-cassany/

Cervantes López, Miriam Janet, \& Llanes Castillo, Arturo, \& Peña Maldonado, Alma Alicia, \& Cruz Casados, Jaime (2020). Estrategias para potenciar el aprendizaje y el rendimiento académico en estudiantes universitarios. Revista Venezolana de Gerencia, 25(90),579-594. https://www.redalyc.org/articulo.oa?id=29063559011

Cumbajín, I. (2018). Estrategias Didácticas para Reducir el Filtro Afectivo en el Aprendizaje del Idioma Inglés, en la Unidad Educativa José Mejía Lequerica. Quito: Pontifica Universidad Católica del Ecuador.

Díaz, E. (2011). Enfoques de aprendizaje y niveles de comprensión. Colombia: Córdoba.

Fabregat, B. (2015). La inteligencia emocional en el aula de lengua inglesa. Máster universitario en comunicación intercultural y enseñanza de lenguas. Universitat Jaume I. España. 
Fernández, L. (2015). La inteligencia emocional en el aula Bilingüe. Universidad de Valladolid. España.

García, J. (2012). La educación emocional, su importancia en el proceso de aprendizaje. Revista Educación.

Gutiérrez Ramírez, Maricel, \& Landeros Falcón, Ignacio Ariel (2010). Importancia del lenguaje en el contexto de la aldea global. Horizontes Educacionales, 15(1),95-107. ISSN: 0717-2141. https://www.redalyc.org/ pdf/979/97916218008.pdf

Lojano, A. (2017) cómo influye la autoestima en el rendimiento académico delos estudiantes de tercer año de educación básica de la escuela fisco misional Cuenca en el área de matemáticas, en el periodo 20152016. p. 23-38

Lozano, Luis \& García-Cueto, Eduardo \& álvaro, Pedro \& Asturias, Colegio. (2000). Relación entre motivación y aprendizaje. Psicothema. 12. 344-347.

Martínez, M. (2018). La importancia de introducir la competencia intercultural en la educación superior: Propuesta de actividades prácticas. Revista Electrónica Educare, 22(1). https://www.redalyc.org/jatsRepo/1941/194154980002/html/index.html

Montes de Oca, R. (2017). Autoestima e idioma inglés: una primera discusión. https://www.redalyc.org/articulo. oa?id=44029106

Moreno, K. (2017). Factores emocionales que influyen en filtro afectivo de los estudiantes de inglés como idioma extranjero, una revisión sistemática. https://www.revistaespirales.com/index.php/es/article/view/19/0

Ospina R., J. (2006). La motivación, motor del aprendizaje. Revista Ciencias de la Salud. 4. 158-160.

Parra F., Keila N. (2014). El docente y el uso de la mediación en los procesos de enseñanza y aprendizaje. Revista de Investigación, 38(83),155-180. ISSN: 0798- 0329. https://www.redalyc.org/pdf/3761/376140398009. pdf

Sáiz Manzanares, M.C., y Queiruga, M. A. (2018). Evaluación de estrategias metacognitivas: aplicación de métodos online. Revista de Psicología y Educación,13(1), 33-45, https://doi.org/10.23923/rpye2018.01.156

Sánchez, I. (2017). Aspectos psicológicos que pueden influir en la adquisición de una lengua extranjera. Revista Didáctica de Lengua y Literatura.

Schunk, D. (1997). Teorías del aprendizaje. Pearson educación.

Shehri, H. (2012). Los modelos de adquisición y enseñanza de una segunda lengua. Avances en Supervisión Educativa.

Vaello O., J. (2005): Habilidades sociales en el aula. Santillana. 
Valenzuela C., F., Pérez Villalobos, M. Victoria, Bustos, Claudio, \& Salcedo Lagos, Pedro. (2018). Cambios en el concepto aprendizaje de estudiantes de pedagogía: análisis de disponibilidad léxica y grafos. Estudios filológicos, (61), 143-173. https://dx.doi.org/10.4067/S0071-17132018000100143

Yánez, P. (2015). El proceso de aprendizaje: fases y elementos fundamentales. Barcelona: Revista San Gregorio. 
Copyright (c) 2021 Nancy Cristina Uquillas Jaramillo y Karen Stephany Córdova Vera

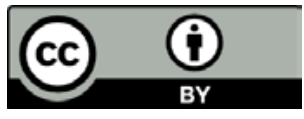

Este texto está protegido bajo una licencia internacional Creative Commons 4.0.

Usted es libre para Compartir-copiar y redistribuir el material en cualquier medio o formato

- y Adaptar el documento - remezclar, transformar y crear a partir del material-para cualquier propósito, incluso para fines comerciales, siempre que cumpla las condiciones de Atribución. Usted debe dar crédito a la obra original de manera adecuada, proporcionar un enlace a la licencia, e indicar si se han realizado cambios. Puede hacerlo en cualquier forma razonable, pero no de forma tal que sugiera que tiene el apoyo del licenciante o lo recibe por el uso que hace de la obra.

$\underline{\text { Resumen de licencia - Texto completo de la licencia }}$ 